\title{
Morphometric study of the extraocular muscles in Thai population
}

\author{
N. Surawatsatien, Y. Papassornsiri, V. Chentanez \\ Department of Anatomy, Faculty of Medicine, King Chulalongkorn Memorial Hospital, Chulalongkorn University, \\ Bangkok, Thailand
}

[Received: 17 January 2017; Accepted: 21 February 2017]

\begin{abstract}
Morphology of the extraocular muscles plays an important role in many ophthalmological surgeries and diagnostic imaging studies. Detailed understanding of length, width, point of insertion and the relationships between these muscles could be of significance for successful surgical outcomes. Forty-six orbital contents were extracted from formaldehyde-embalmed cadavers. Dissection was performed to reveal the extraocular muscles from their origins to insertions on the sclera. Muscle length, muscle width, distance from point of insertion to the sclerocorneal limbus, relationship between the superior oblique and superior rectus and between the inferior oblique and lateral rectus were measured. The lateral rectus and superior rectus were the longest muscles. The insertion of the medial rectus muscle was closest to the sclerocorneal limbus while the inferior rectus, lateral rectus and superior rectus insertions were progressively further apart. Only the length of the inferior rectus and medial rectus were significantly different between males and females. The distances between the superior oblique and superior rectus and between the inferior oblique and lateral rectus were slightly greater than in previous study. A direct comparison of these parameters between ethnic groups is still needed for an improvement of the procedural outcomes. (Folia Morphol 2017; 76, 4: 635-641)
\end{abstract}

Key words: extraocular muscles, morphology, Thai, superior rectus, inferior rectus, lateral rectus, medial rectus, superior oblique, inferior oblique

\section{INTRODUCTION}

The extraocular muscles (EOM) play an important role in both voluntary and involuntary eye movements which are essential for binocular vision $[3,17]$. Knowledge of the morphometric parameters of these muscles plays a fundamental role in many ophthalmological surgeries, especially in strabismus surgery. Detailed information of length, width, specific point of insertion and relationship between these muscles are vital to successful surgery both in terms of results and complications such as under or over correction and anterior segment ischaemia [7]. This knowledge can also be used in many imaging studies to diagnose many diseases such as Graves' disease, intraorbital tumours, acromegaly, arteriovenous malformation, endophthalmitis, and intraorbital trauma [10]. Blake et al. [2] reported that the orbital structures were different between races and ethnic groups. Several previous morphometric studies of the EOM in specific population by various techniques also reported different results $[1,8,9,11,16,19]$. There has been an indirect imaging morphometric study of the EOM

Address for correspondence: V. Chentanez MD, PhD, Department of Anatomy, Faculty of Medicine, King Chulalongkorn Memorial Hospital, Chulalongkorn University, Bangkok 10330, Thailand, tel: 66-860701084, e-mail: fmedvct@gmail.com 


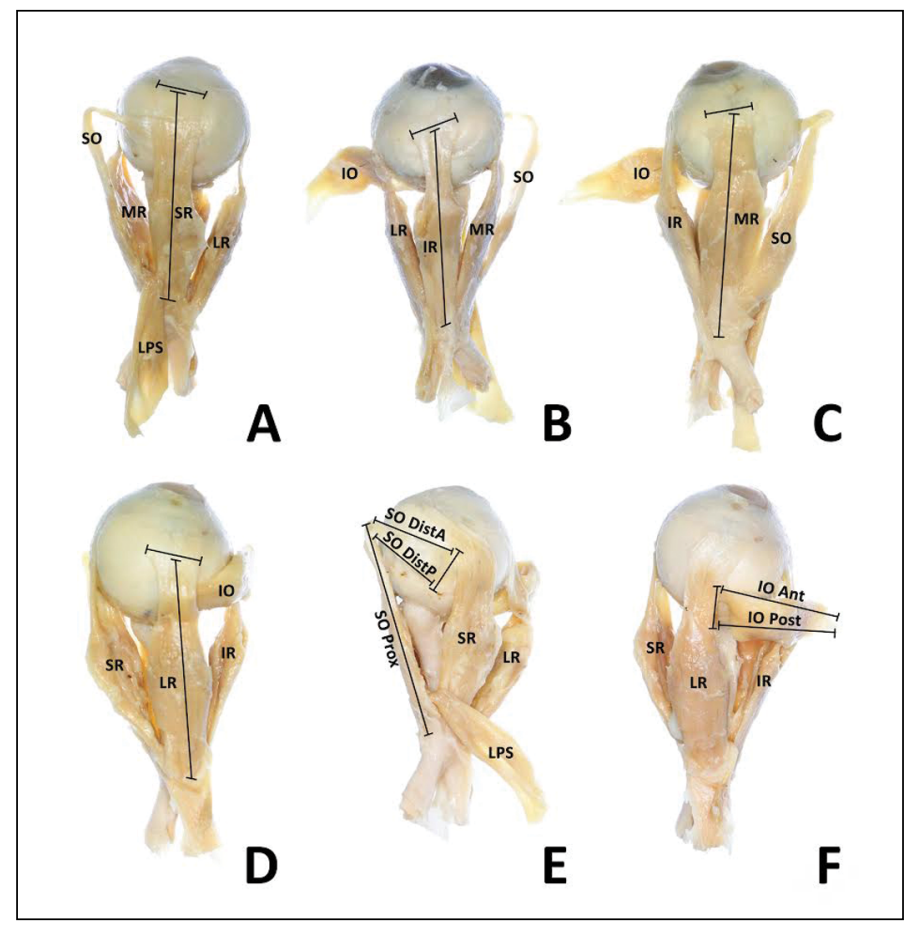

Figure 1. Measurement of length and width of extraocular muscles; A. Superior rectus muscle; B. Inferior rectus muscle; C. Medial rectus muscle; D. Lateral rectus muscle; E. Superior oblique muscle; Prox - proximal segment; Dist A — distal anterior; Dist P — distal posterior; F. Inferior oblique muscle; Ant — Anterior; Post — posterior; SR superior rectus; IR - inferior rectus; $\mathrm{MR}$ - medial rectus; LR — lateral rectus; SO — superior oblique; 10 — inferior oblique. in Thai population [10]; however, to the best of our knowledge, there is no direct cadaveric study available. Therefore, this study aimed to analyse the morphometric parameters of the EOM, their relationships, possible anatomical variations and compare these parameter differences between genders.

\section{MATERIALS AND METHODS}

Orbital contents were extracted from 25 male and 21 female formaldehyde-embalmed cadavers (age range 41-99) provided by the Department of Anatomy, Faculty of Medicine, Chulalongkorn University. All cadavers had no history of orbital trauma or surgery. Muscles and connective tissue of the face and skull, calvarias and intracranial contents were removed as a part of the medical student anatomy class. Superior orbitotomy with facial and intracranial approach were done by researchers to extract the orbital content which was then placed in a mold to facilitate the dissection. After completing dissection of each globe, a standardised digital vernier calliper (Baker ${ }^{\mathrm{TM}}$ EC-10; range 6"/150 mm, resolution $0.0005 " / 0.01 \mathrm{~mm}$ ) was used to measure each parameter. The length of each rectus muscle, superior oblique (SO) proximal segment (SO Prox), SO anterior distal segment (SO DistA), SO posterior distal segment (SO DistP), inferior oblique (IO) anterior segment (IO Ant) and the 10 posterior segment (IO Post), width at the point of insertion of each muscle and the dis- tance between the insertion of each muscle to the sclerocorneal limbus were measured. Each parameter was measured twice and the average number was reported. To ensure consistency, the same digital vernier calliper was used and the same investigator recorded each parameter twice. For intra-observer reliability, the second measurement was taken after a 2-week interval. The method of measurement of each parameter including the points of measurement followed the definition in Villarreal-Silva et al. [19] (Figs. 1-3).

\section{Statistical analysis}

Data were calculated and analysed by Microsoft Excel 2013 and IBM SPSS Version 22. Mean and standard deviation for each parameter were obtained. Results were then separated into male and female groups. Shapiro-Wilk test of normality was used to determine the distribution of the sample population. Comparisons of the parameters between male and female subjects were done using unpaired t-test in parameter with normally distributed data and MannWhitney $\mathrm{U}$ test in parameters with non-normally distributed data.

\section{RESULTS}

\section{Lengths of the EOM}

The results of length of the rectus muscles revealed that the lateral rectus (LR) was the longest 

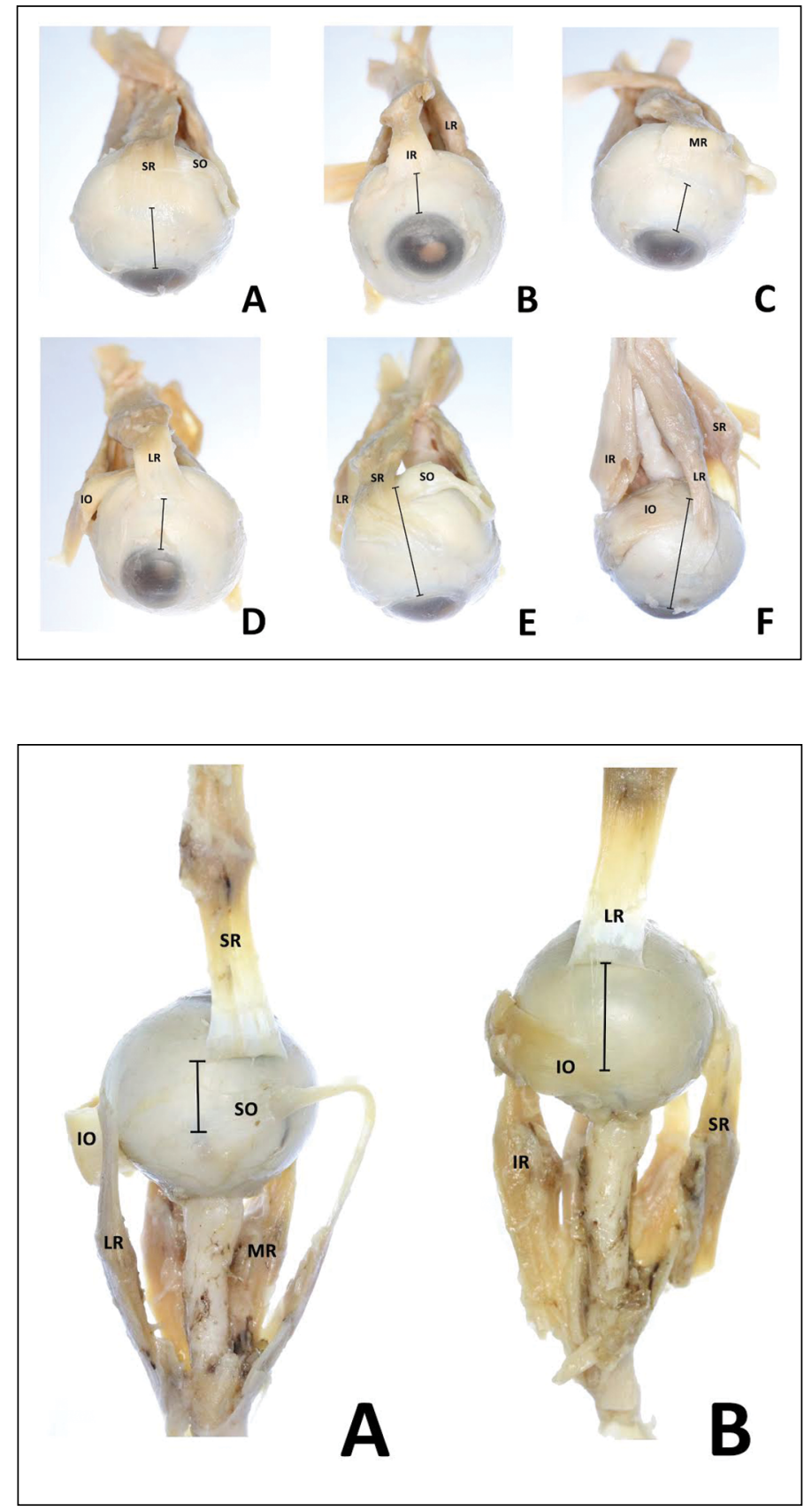

Figure 2. Distance between point of insertion of the extraocular muscles and sclerocorneal limbus; A. Superior rectus muscle; B. Inferior rectus muscle; C. Medial rectus muscle; D. Lateral rectus muscle; E. Superior oblique muscle; $\mathbf{F}$ Inferior oblique muscle; SR - superior rectus; IR — inferior rectus; MR - medial rectus; LR — lateral rectus; $\mathrm{SO}$ - superior oblique; 10 — inferior oblique.

Figure 3. Distance between oblique and rectus muscles insertion; A. Between superior oblique and superior rectus muscles; B. Between inferior oblique and lateral rectus muscles; SR - superior rectus; IR - inferior rectus; $\mathrm{MR}$ - medial rectus; LR — lateral rectus; SO — superior oblique; 10 - inferior oblique.

with a mean length of $45.71 \pm 2.05 \mathrm{~mm}$. This was followed in numerical order by the superior rectus (SR), inferior rectus (IR) and then the medial rectus (MR) with a mean length of $39.44 \pm 2.47 \mathrm{~mm}$. The mean length of SO Prox was $40.85 \pm 2.25 \mathrm{~mm}$ while the mean lengths of SO DistA and SO DistP were $19.04 \pm 1.27$ and $17.06 \pm 1.04 \mathrm{~mm}$, respectively. The 10 was the shortest with a mean length of $27.09 \pm 2.26 \mathrm{~mm}$ for $\mathrm{IO}$ Ant and $28.01 \pm 2.93 \mathrm{~mm}$ for $\mathrm{IO}$ Post (Table 1). Lengths of all rectus muscles, SO Prox, IO Ant and IO Post were normally distributed while the lengths of the SO DistA and SO DistP were not normally distributed. A comparison between gen- ders revealed that only the IR and MR lengths were significantly different $(p=0.016$ and $p=0.037$, respectively) (Table 2 ).

\section{Widths of the EOM}

The SR was the widest EOM with a mean length of $9.64 \pm 0.94 \mathrm{~mm}$. This was followed in numerical order by the IO, SO, MR, LR, and then the IR with a mean length of $8.31 \pm 2.09 \mathrm{~mm}$ (Table 1). Widths of the IR, MR, LR and IO were normally distributed while widths of the SR and SO were not normally distributed. The widths between genders had not statistically significant difference (Table 2). 
Table 1. The lengths, widths and insertions $[\mathrm{mm}]$ of the extraocular muscles

\begin{tabular}{|c|c|c|c|c|}
\hline \multicolumn{2}{|c|}{ Muscles } & Length & Width & Insertion \\
\hline \multicolumn{2}{|l|}{ SR } & $42.85 \pm 2.59$ & $9.64 \pm 0.94$ & $7.35 \pm 0.39$ \\
\hline \multicolumn{2}{|l|}{ IR } & $41.30 \pm 2.09$ & $8.31 \pm 2.09$ & $6.59 \pm 0.34$ \\
\hline \multicolumn{2}{|l|}{ MR } & $39.44 \pm 2.47$ & $9.17 \pm 0.90$ & $5.70 \pm 0.41$ \\
\hline \multicolumn{2}{|l|}{ LR } & $45.71 \pm 2.05$ & $8.61 \pm 0.78$ & $6.94 \pm 0.49$ \\
\hline \multirow[t]{3}{*}{ SO } & Prox & $40.85 \pm 2.25$ & & \\
\hline & DistA & $19.04 \pm 1.27$ & $9.27 \pm 1.19$ & $16.88 \pm 0.98$ \\
\hline & DistP & $17.06 \pm 1.04$ & & \\
\hline \multirow[t]{2}{*}{10} & Ant & $27.09 \pm 2.26$ & \multirow{2}{*}{$9.52 \pm 1.01$} & \multirow{2}{*}{$18.38 \pm 1.09$} \\
\hline & Post & $28.01 \pm 2.93$ & & \\
\hline
\end{tabular}

SR — superior rectus; IR — inferior rectus; MR — medial rectus; LR — lateral rectus; SO — superior oblique; 10 - inferior oblique; Prox — proximal segment; DistA — distal anterior; DistP — distal posterior; Ant — Anterior; Post — posterior

Table 2. Comparison of the lengths, widths and insertions $[\mathrm{mm}]$ of the extraocular muscles between males and females

\begin{tabular}{|c|c|c|c|c|c|c|c|c|c|c|}
\hline \multicolumn{2}{|c|}{ Muscles } & \multicolumn{3}{|c|}{ Length } & \multicolumn{3}{|c|}{ Width } & \multicolumn{3}{|c|}{ Insertion } \\
\hline & & Males & Females & $\mathbf{p}$ & Males & Females & $\mathbf{p}$ & Males & Females & p \\
\hline SR & & $42.96 \pm 2.86$ & $42.71 \pm 2.28$ & 0.742 & $90.72 \pm 10.00$ & $9.54 \pm 0.88$ & 0.938 & $70.41 \pm 00.40$ & $7.27 \pm 0.39$ & 0.232 \\
\hline $\mathbb{I R}$ & & $41.95 \pm 1.83$ & $40.49 \pm 2.14$ & $0.016^{*}$ & $80.47 \pm 00.88$ & $8.12 \pm 0.66$ & 0.143 & $60.58 \pm 00.37$ & $6.61 \pm 0.32$ & 0.770 \\
\hline MR & & $40.13 \pm 2.35$ & $38.61 \pm 2.41$ & $0.037^{*}$ & $90.28 \pm 00.97$ & $9.05 \pm 0.81$ & 0.379 & $50.75 \pm 00.35$ & $5.62 \pm 0.46$ & 0.279 \\
\hline LR & & $46.23 \pm 1.82$ & $45.08 \pm 2.17$ & 0.056 & $80.67 \pm 00.69$ & $8.55 \pm 0.89$ & 0.605 & $60.97 \pm 00.47$ & $6.91 \pm 0.52$ & 0.691 \\
\hline \multirow[t]{3}{*}{ SO } & Prox & $41.22 \pm 2.17$ & $400.42 \pm 20.31$ & 0.233 & & & & & & \\
\hline & DistA & $19.32 \pm 1.31$ & $180.72 \pm 10.17$ & 0.076 & $9.19 \pm 0.97$ & $90.36 \pm 10.43$ & 0.256 & $16.88 \pm 1.20$ & $160.88 \pm 00.67$ & 0.930 \\
\hline & DistP & $17.21 \pm 0.88$ & $160.89 \pm 10.19$ & 0.225 & & & & & & \\
\hline \multirow[t]{2}{*}{10} & Ant & $26.84 \pm 2.67$ & $270.39 \pm 10.64$ & 0.416 & & & & & & 0 \\
\hline & Post & $27.52 \pm 3.40$ & $280.59 \pm 20.17$ & 0.223 & 0 & & $t$ & & & \\
\hline
\end{tabular}

SR — superior rectus; IR — inferior rectus; $M R$ - medial rectus; LR — lateral rectus; SO — superior oblique; 10 — inferior oblique; Prox — proximal segment; DistA — distal anterior; DistP — distal posterior; Ant — Anterior; Post — posterior; * ${ }^{*}$ tatistically significant

Table 3. Comparison of the relationships of the oblique and rectus muscles between males and females

\begin{tabular}{lcccc}
\hline Parameters & Total & \multicolumn{3}{c}{ Distance $(\mathrm{mm})$} \\
\cline { 3 - 5 } & & Male & Female & $\mathbf{p}$ \\
\hline SO-SR & $11.00 \pm 0.90$ & $11.01 \pm 0.93$ & $10.99 \pm 0.87$ & 0.933 \\
IO-LR & $13.40 \pm 0.90$ & $13.47 \pm 0.96$ & $13.32 \pm 0.84$ & 0.591 \\
\hline
\end{tabular}

SR — superior rectus; LR — lateral rectus; $S 0$ - superior oblique; 10 - inferior oblique

\section{Insertions of the EOM}

The insertions of the MR, IR, LR, and SR were located at $5.70 \pm 0.41 \mathrm{~mm}, 6.59 \pm 0.34 \mathrm{~mm}, 6.94 \pm$ $\pm 0.49 \mathrm{~mm}$ and $7.35 \pm 0.39 \mathrm{~mm}$ from the sclerocorneal limbus, respectively. The insertions of the SO and $\mathrm{IO}$ were located at $16.88 \pm 0.98 \mathrm{~mm}$ and $18.38 \pm$ $\pm 1.09 \mathrm{~mm}$, respectively (Table 1 ). The insertions of the EOM were normally distributed except for the SO insertion. There was no significant difference of the insertions between genders (Table 2).

\section{Relationships of oblique and rectus muscle}

The SO-SR and IO-IR distances were normally distributed with mean distances of $11.00 \pm 0.90 \mathrm{~mm}$ and $13.40 \pm 0.90 \mathrm{~mm}$, respectively. There was no statistically significant difference in these relationships between genders (Table 3).

\section{DISCUSSION}

The result of this study coincides with a study by Villarreal-Silva et al. [19] that revealed LR and SR were the longest rectus muscles, while MR and IR were the shortest (Table 4). The length of each muscle could be used in determining the dimension of the muscle 
Table 4. Comparison of the extraocular muscles lengths [mm] from this study and from two previous studies $[15,19]$

\begin{tabular}{|c|c|c|c|c|}
\hline & & Villarreal et al. [19] & Ridyard [15] & Current study \\
\hline \multicolumn{2}{|l|}{$\mathrm{N}$} & 20 & 18 & 46 \\
\hline \multicolumn{2}{|c|}{ Measurement type } & $\begin{array}{l}\text { Direct measurement } \\
\text { (dissection) }\end{array}$ & $\begin{array}{l}\text { Direct measurement } \\
\text { (dissection) }\end{array}$ & $\begin{array}{l}\text { Direct measurement } \\
\text { (dissection) }\end{array}$ \\
\hline \multicolumn{2}{|l|}{ SR } & $37.46 \pm 3.10$ & $38.20 \pm 4.10$ & $42.85 \pm 2.59$ \\
\hline \multicolumn{2}{|l|}{$\mathbb{R}$} & $34.89 \pm 3.54$ & $37.20 \pm 2.40$ & $41.30 \pm 2.09$ \\
\hline \multicolumn{2}{|c|}{ MR } & $35.22 \pm 3.45$ & $38.50 \pm 3.10$ & $39.44 \pm 2.47$ \\
\hline \multicolumn{2}{|l|}{$\mathrm{LR}$} & $38.81 \pm 3.00$ & $38.40 \pm 2.40$ & $45.71 \pm 2.05$ \\
\hline \multirow[t]{3}{*}{ SO } & Prox & $33.50 \pm 2.44$ & & $40.85 \pm 2.25$ \\
\hline & DistA & $20.61 \pm 1.98$ & $39.20 \pm 4.50$ & $19.04 \pm 1.27$ \\
\hline & DistP & $18.25 \pm 2.51$ & & $17.06 \pm 1.04$ \\
\hline \multirow[t]{2}{*}{10} & Ant & $27.40 \pm 3.30$ & \multirow{2}{*}{$22.50 \pm 4.40$} & $27.09 \pm 2.26$ \\
\hline & Post & $30.08 \pm 3.75$ & & $28.01 \pm 2.93$ \\
\hline
\end{tabular}

SR — superior rectus; IR — inferior rectus; MR — medial rectus; LR — lateral rectus; SO — superior oblique; 10 - inferior oblique; Prox — proximal segment; DistA — distal anterior; DistP — distal posterior; Ant - Anterior; Post — posterior

Table 5. Comparison of the extraocular muscles widths or diameters [mm] from this study and from five previous studies $[1,9,10,16,19]$ with different nationality, measurement type and point of measurement

\begin{tabular}{|c|c|c|c|c|c|c|}
\hline & $\begin{array}{l}\text { Villarreal } \\
\text { et al. [19] }\end{array}$ & $\begin{array}{l}\text { Athavale } \\
\text { et al. [1] }\end{array}$ & $\begin{array}{l}\text { Lerdlum } \\
\text { et al. [10] }\end{array}$ & $\begin{array}{c}\text { Shen } \\
\text { et al. [16] }\end{array}$ & $\begin{array}{c}\text { Lee } \\
\text { et al. [9] }\end{array}$ & Current study \\
\hline $\mathrm{N}$ & 20 & 40 & 400 & 160 & 428 & 46 \\
\hline Nationality & Mexican & Indian & Thai & Chinese & Korean & Thai \\
\hline Measurement type & $\begin{array}{c}\text { Direct } \\
\text { (dissection) }\end{array}$ & $\begin{array}{c}\text { Direct } \\
\text { (dissection) }\end{array}$ & CT scan & MRI & CT scan & $\begin{array}{c}\text { Direct } \\
\text { (dissection) }\end{array}$ \\
\hline $\begin{array}{l}\text { Point of } \\
\text { measurement }\end{array}$ & $\begin{array}{c}\text { Point } \\
\text { of insertion }\end{array}$ & $\begin{array}{c}\text { Point } \\
\text { of insertion }\end{array}$ & $\begin{array}{l}\text { Widest } \\
\text { point }\end{array}$ & $\begin{array}{l}\text { Widest } \\
\text { point }\end{array}$ & $\begin{array}{l}\text { Widest } \\
\text { point }\end{array}$ & $\begin{array}{c}\text { Point } \\
\text { of insertion }\end{array}$ \\
\hline LPS & $12.94 \pm 2.66$ & - & $3.80 \pm 1.40^{*}$ & $4.80^{*}$ & $4.40 \pm 1.40^{*}$ & - \\
\hline SR & $8.68 \pm 1.51$ & $9.11 \pm 2.10$ & & $(2.60-7.00)$ & & $9.64 \pm 0.94$ \\
\hline $\mathbb{R}$ & $6.85 \pm 0.70$ & $8.72 \pm 2.56$ & $4.00 \pm 1.40$ & $5.40(3.40-7.40)$ & $4.10 \pm 1.60$ & $8.31 \pm 2.09$ \\
\hline MR & $8.13 \pm 0.84$ & $9.60 \pm 2.24$ & $3.70 \pm 0.90$ & $5.10(3.30-6.90)$ & $3.70 \pm 1.50$ & $9.17 \pm 0.90$ \\
\hline $\mathrm{LR}$ & $7.76 \pm 1.40$ & $8.90 \pm 2.30$ & $3.60 \pm 1.20$ & $4.50(2.70-6.30)$ & $3.40 \pm 1.30$ & $8.61 \pm 0.78$ \\
\hline so & $7.10 \pm 1.47$ & $8.38 \pm 3.36$ & - & - & - & $9.27 \pm 1.19$ \\
\hline 10 & $9.23 \pm 0.90$ & $7.90 \pm 1.90$ & - & - & - & $9.52 \pm 1.01$ \\
\hline
\end{tabular}

*The superior rectus and the levator palpebrae superioris muscle were measured together as a single muscle group because they could not be reliably distinguished from each other; CT — computed tomography; MRI — magnetic resonance imaging; LPS — levator palpebrae superioris; SR — superior rectus; IR — inferior rectus; MR — medial rectus; LR — lateral rectus; $\mathrm{SO}$ - superior oblique; 10 - inferior oblique

cone surrounding the globe [19]. From these findings, it could be concluded that the lateral and superior segments of the muscle cone were larger thus in surgery, approaching through the space between the $S R$ and $L R$ was considered to be safer.

A reference width of the rectus muscles insertion is widely used in a procedure to reduce strength of the rectus muscles by resecting the muscle fibres in patients with esotropia or exotropia [3]. In compari- son with other studies $[1,9,10,16,19]$, the widths of the EOM from direct measurement were wider than those measured with computed tomography scan or magnetic resonance imaging (Table 5). VillarrealSilva et al. [19] reported that the differences in width between the SR and IR and between the LR and MR were $1.83 \mathrm{~mm}$ and $0.37 \mathrm{~mm}$, respectively, whereas differences reported in this study were $1.33 \mathrm{~mm}$ and $0.56 \mathrm{~mm}$, respectively. The differences between these 
Table 6. Comparison of the extraocular muscles insertions [mm] from this study and from four previous studies $[1,8,18,19]$ with different measurement type

\begin{tabular}{lcccccc}
\hline & Villarreal et al. [19] & Athavale et al. [1] & Tamburrelli et al. [18] & Lai et al. [8] & Current study \\
\hline $\mathrm{N}$ & 20 & 40 & 19 & & 60 & 46 \\
Measurement type & $\begin{array}{c}\text { Direct } \\
\text { (dissection) }\end{array}$ & $\begin{array}{c}\text { Direct } \\
\text { (dissection) }\end{array}$ & $\begin{array}{c}\text { Ultrasound } \\
\text { (pre-op) }\end{array}$ & $\begin{array}{c}\text { Direct } \\
\text { (intra-op) }\end{array}$ & $\begin{array}{c}\text { Direct } \\
\text { (intra-op) }\end{array}$ & $\begin{array}{c}\text { Direct } \\
\text { (dissection) }\end{array}$ \\
SR & $7.52 \pm 1.13$ & $8.74 \pm 1.66$ & - & - & $6.80 \pm 0.70$ & $7.35 \pm 0.39$ \\
IR & $6.80 \pm 0.96$ & $8.06 \pm 2.06$ & - & - & $6.00 \pm 0.80$ & $6.59 \pm 0.34$ \\
MR & $5.99 \pm 0.80$ & $7.34 \pm 1.56$ & $5.61 \pm 0.62$ & $5.46 \pm 0.76$ & $5.20 \pm 0.90$ & $5.70 \pm 0.41$ \\
LR & $6.77 \pm 0.61$ & $8.71 \pm 2.54$ & $5.76 \pm 0.60$ & $6.25 \pm 0.51$ & $6.30 \pm 0.90$ & $6.94 \pm 0.49$ \\
SO & $15.92 \pm 1.20$ & - & - & - & - & $16.88 \pm 0.98$ \\
IO & $17.83 \pm 1.07$ & - & - & - & - & $18.38 \pm 1.09$ \\
\hline
\end{tabular}

SR — superior rectus; IR — inferior rectus; MR — medial rectus; LR — lateral rectus; SO — superior oblique; 10 — inferior oblique

antagonist tendons could be important in the vertical resection or transposition operation in patients with CN VI palsy [3].

The strabismus surgery also requires detailed knowledge of the point of insertion of each EOM. Fuchs [6] described the characteristic of the EOM insertion points as non-parallel to the horizontal meridian of the globe. As mentioned above, the insertions of the MR, IR, LR and SR in this study were located at $5.7 \mathrm{~mm}, 6.69 \mathrm{~mm}, 6.94 \mathrm{~mm}$ and $7.35 \mathrm{~mm}$ from the sclerocorneal limbus, respectively. Results of this study concurred with previous studies $[1,8,18,19]$ and the original measurement by P.J. Tillaux which confirmed that the insertion of the MR was the closest one to the sclerocorneal limbus while the IR, LR and SR insertions progressively became further apart [4] (Table 6).

Recent studies established differences in these morphometric measurements between genders. The studies of Özgenand and Ariyurek [12] and Özgen and Aydingöz [13] agreed that the diameters of the EOM were larger in males than in females. While Ogbole et al. [11] found this difference only in the superior muscle group which consisted of the SR and levator palpebrae superioris. As previously mentioned, this study revealed that only the length of the IR and MR was significantly different between males and females. In contrast, Lerdlum et al. [10], Shen et al. [16] and Lee et al. [9] found no significant difference in any parameters between sexes.

This relationship between the SO and SR could be useful during a surgical procedure on the SO. Although this current study used the same measurement parameter as the Villarreal-Silva study [19], the result was slightly greater than the original. von Noorden and
Campos [20] also studied this relationship; however, they used different parameters so the results could not be compared. The IO has recently become the muscle of interest in the implantation of trans scleral drug delivery devices which is a novel treatment option for diabetic maculopathy and age-related macular degeneration [5]. Yalçin and Ozan [21] identified the variations of 10 insertion to be divided into multiple tendons in $91.7 \%$ of the study population while Paik and Shin [14] found these variations only in $45 \%$ of the embalmed specimens but none in fresh subjects. This study found no such variations. The relationship of the IO and LR has been studied by Villarreal-Silva et al. [19], Feng et al. [5] and Paik and Shin [14]; however, due to the fact that each study developed its own measurement parameter, results could not be compared. The current study uses the same parameter as Villarreal-Silva et al. [19] and the result of relationship between IO and LR was slightly greater than the Villarreal-Silva's study [19].

\section{Limitations of the study}

Some study limitations include the use of embalmed cadavers which might yield different results from fresh cadavers as reported by Paik and Shin [14]. Anatomy tutorial cadavers were used in this study. Thus the orbital contents could be retrieved from only one side as the other side was used by medical students. Therefore, this study could not compare parameter differences between left and right orbits.

\section{Acknowledgements}

The authors would like to show our sincere appreciation to all those who have donated their bodies to science for the advancement of medical research. 
Much kind thanks to the Department of Anatomy, Faculty of Medicine, Chulalongkorn University and its staff for study assistance.

\section{REFERENCES}

1. Athavale S, Kotgirwar S, Lalwani R. Rectus and oblique muscles of eyeball: a morphometric study of Indian population. Anat Cell Biol. 2015; 48(3): 201-204, doi: 10.5115/ /acb.2015.48.3.201, indexed in Pubmed: 26417480.

2. Blake CR, Lai WW, Edward DP. Racial and ethnic differences in ocular anatomy. Int Ophthalmol Clin. 2003; 43(4): 9-25, indexed in Pubmed: 14574198.

3. Bowling B. Strabismus. In: Kanski's clinical ophthalmology: a systemic approach, 8th Ed. Elsevier, Atlanta 2016: 728-771.

4. Dahlmann-Noor A. P. J. Tillaux: Of the Way the Muscles Insert onto the Sclera. Strabismus. 2008; 16(4): 174-175, doi: 10.1080/09273970802637558.

5. Feng $X$, Pilon K, Yaacobi $Y$, et al. Extraocular muscle insertions relative to the fovea and optic nerve: humans and rhesus macaque. Invest Ophthalmol Vis Sci. 2005; 46(10): 3493-3496, doi: 10.1167/iovs.05-0283, indexed in Pubmed: 16186324.

6. Fuchs E. Beiträge zur normalen Anatomie des Augapfels. Graefes Arch Ophthalmol. 1894; 30: 1-65.

7. Hong S, Chang $\mathrm{YH}$, Han $\mathrm{SH}$, et al. Effect of full tendon transposition augmented with posterior intermuscular suture for paralytic strabismus. Am J Ophthalmol. 2005; 140(3): 477-483, doi: 10.1016/j.ajo.2005.04.028, indexed in Pubmed: 16084787.

8. Lai $\mathrm{YH}, \mathrm{Wu} \mathrm{WC}$, Wang $\mathrm{HZ}$, et al. Extraocular muscle insertion positions and outcomes of strabismus surgery: correlation analysis and anatomical comparison of Western and Chinese populations. Br J Ophthalmol. 2012; 96(5): 679-682, doi: 10.1136/bjophthalmol-2011-300632, indexed in Pubmed: 22241925.

9. Lee JS, Lim DW, Lee SH, et al. Normative measurements of Korean orbital structures revealed by computerized tomography. Acta Ophthalmol Scand. 2001; 79(2): 197-200, indexed in Pubmed: 11284763.

10. Lerdlum S, Boonsirikamchai P, Setsakol E. Normal measurements of extraocular muscle using computed tomography. J Med Assoc Thai. 2007; 90(2): 307-312, indexed in Pubmed: 17375636.
11. Ogbole Gl, Ogun OA, Olusumade D. Computed tomography measurement of extra-ocular muscle diameters in a population of normal patients in a tertiary hospital in Nigeria. Afr J Med Med Sci. 2014; 43(3): 245-250, indexed in Pubmed: 26223143.

12. Ozgen A, Ariyurek M. Normative measurements of orbital structures using CT. AJR Am J Roentgenol. 1998; 170(4): 1093-1096, doi: 10.2214/ajr.170.4.9530066, indexed in Pubmed: 9530066.

13. Ozgen A, Aydingöz U. Normative measurements of orbital structures using MRI. J Comput Assist Tomogr. 2000; 24(3): 493-496, indexed in Pubmed: 10864092.

14. Paik DJ, Shin SY. An anatomical study of the inferior oblique muscle: The embalmed cadaver vs the fresh cadaver. Am J Ophthalmol. 2009; 147(3): 544-549.e1, doi: 10.1016/j. ajo.2008.09.012, indexed in Pubmed: 19054494.

15. Ridyard E. Extraocular muscles: variation in their anatomy, length and cross-sectional diameter. Int J Anat Res. 2015; 3(3): 1198-1206, doi: 10.16965/ijar.2015.164.

16. Shen $S$, Fong KS, Wong HB, et al. Normative measurements of the Chinese extraocular musculature by high-field magnetic resonance imaging. Invest Ophthalmol Vis Sci. 2010; 51(2): 631-636, doi: 10.1167/iovs.09-3614, indexed in Pubmed: 19643971.

17. Snell RS. LempMA Movement of the eyeball and extraocular muscles. In: Clinical anatomy of the eye. 2 nd Ed. Blackwell Science, New Jersey 1998: 231-271.

18. Tamburrelli $C$, Salgarello T, Vaiano AS, et al. Ultrasound of the horizontal rectus muscle insertion sites: implications in preoperative assessment of strabismus. Invest Ophthalmol Vis Sci. 2003; 44(2): 618-622, indexed in Pubmed: 12556391.

19. Villarreal-Silva E, Hinojosa AJM, Bazaldua CJJ, et al. A morphometric study of the extraocular muscles. Int J Morphol. 2013; 31(1): 312-320, doi: 10.4067/s071795022013000100049.

20. von Noorden, Campos EC. Summary of the gross anatomy of the extraocular muscles. In: Binocular vision and ocular motility. Theory and management of strabismus. 6th Ed. Mosby, St. Louis 2002: 41-52.

21. Yalçin B, Ozan H. Insertional pattern of the inferior oblique muscle. Am J Ophthalmol. 2005; 139(3): 504-508, doi: 10.1016/j.ajo.2004.10.057, indexed in Pubmed: 15767061. 